\title{
MAPK pathways are involved in the inhibitory effect of berberine hydrochloride on gastric cancer MGC 803 cell proliferation and IL-8 secretion in vitro and in vivo
}

\author{
HONG-LI LI, HUI WU, BEI-BEI ZHANG, HAI-LIAN SHI and XIAO-JUN WU \\ Shanghai Key Laboratory of Complex Prescription, Institute of Chinese Materia Medica, \\ The Ministry of Education Key Laboratory for Standardization of Chinese Medicines, \\ Shanghai University of Traditional Chinese Medicine, Shanghai 201203, P.R. China
}

Received July 15, 2015; Accepted May 20, 2016

DOI: $10.3892 / \mathrm{mmr} .2016 .5361$

\begin{abstract}
Gastric cancer is the second leading cause of cancer-associated mortality worldwide. This investigation aimed to identify whether the mitogen-activated protein kinase (MAPK) signaling pathways are involved in the inhibitory effect of berberine hydrochloride (BER) on MGC 803 cells in vitro and in vivo. BER time- and dose-dependently inhibited proliferation of MGC 803 cells. It also suppressed tumorigenesis in nude mice xenografted with MGC 803 cells. Additionally, BER reduced interleukin-8 (IL-8) secretion in vitro and in vivo. Further investigation demonstrated that inactivation of p38 MAPK, extracellular-signal regulated kinase 1/2 and c-Jun N-terminal kinase by BER contributed to the decreased proliferation and tumorigenesis, and the change in IL-8 expression levels. However, there was no significant synergistic inhibitory effect of combined BER and evodiamine (EVO) treatment on tumorigenesis, and BER reduced the upregulation of IL-8 induced by EVO in vivo. The results of the current study suggested that BER may be an effective and safe drug candidate for treating gastric cancer via modulation of the MAPK signaling pathways.
\end{abstract}

\section{Introduction}

Gastric cancer has high metastasis and recurrence rates following curative resection, and is the second leading cause

Correspondence to: Dr Hai-Lian Shi or Professor Xiao-Jun Wu, Shanghai Key Laboratory of Complex Prescription, Institute of Chinese Materia Medica, The Ministry of Education Key Laboratory for Standardization of Chinese Medicines, Shanghai University of Traditional Chinese Medicine, 1200 Cailun Road, Zhangjiang Hi-Tech Park, Shanghai 201203, P.R. China

E-mail: shihailian2003@163.com

E-mail: xiaojunwu320@126.com

Key words: gastric cancer, berberine hydrochloride, evodiamine, mitogen-activated protein kinase, interleukin- 8 of cancer-associated mortality worldwide (1-3). Indeed, the majority of gastric cancer cases are identified at the advanced stages and often develop recurrence following curative resection. Thus, the poor prognosis increases the importance of chemotherapy for treating gastric cancer (4). However, in the clinic, chemotherapy drugs often cause serious side-effects, including immunosuppression, gastrointestinal toxicity and body weakness $(5,6)$. Thus, chemotherapeutic drug candidates derived from natural compounds with low toxicity and low adverse effects have attracted increasing attention.

Interleukin-8 (IL-8), a cytokine of the CXC chemokine family (7), is highly expressed in numerous tumor tissues (8). Accumulating evidence has indicated that overexpression of IL-8 is closely associated with increased adhesion and invasion of human gastric cancer cells, whereas inhibition of IL-8 expression reduces relevant risks (7-10). Accordingly, chemical compounds targeting IL- 8 may be useful for controlling the metastasis of gastric cancer.

Berberine hydrochloride (BER), a major active alkaloid molecule isolated from Coptis Chinensis Franch.(Huanglian), is typically used to treat infectious gastrointestinal diseases and bacterial diarrhea in the clinic. Previous studies demonstrated that BER exerts anti-tumor activity against various types of cancer cells, including human hepatocellular carcinoma (SMMC-7721 cells), gastric cancer (AGS cells, SGC 7901 cells and BGC-823 cells) and colorectal cancer (SW620 cells and LoVo cells) in vitro and in vivo (11-14). Similarly, previous investigation has demonstrated that BER inhibited proliferation and IL-8 expression in AGS cells, a gastric cancer cell line, in vitro $(10,15)$. However, whether BER can prevent gastric cancer development and IL-8 secretion in vivo has not been demonstrated.

BER has previously been demonstrated to modulate mitogen-activated protein kinase (MAPK) signaling pathways, including the extracellular signal-regulated kinase $1 / 2$ (ERK1/2), p38 MAPK and c-Jun N-terminal kinase (JNK) pathways, to exert anti-cancer effects may be cell-type specific. For instance, BER activates MAPKs in human colonic carcinoma cells (16), human hepatoma (HepG2) and non-small cell lung cancer cells (17-19). Whereas in human cervical carcinoma HeLa cells, BER enhances JNK and ERK1/2 
phosphorylation but inhibits p38 MAPK phosphorylation (20). Currently, the effect of BER on MAPK pathways in gastric cancer cells remains poorly understood.

Thus, in present study, the effects of BER on gastric cancer MGC 803 cell proliferation and IL-8 secretion were investigated in vitro and in vivo. Furthermore, the association between MAPK pathway inactivation and the proliferative inhibition of BER, and IL- 8 secretion in MGC 803 cells was also examined. The results may provide a novel and safe strategy for the therapy of gastric cancer using BER.

\section{Materials and methods}

Materials and chemicals. BER (purity, 98\%), evodiamine (EVO; purity, 98\%) and 5-fluorouracil (5-Fu; purity, 98\%) were obtained from Melonepharma Co., Ltd. (Dalian, China). Trypsin and fetal bovine serum (FBS) were obtained from Gibco (Thermo Fisher Scientific, Inc. Waltham, MA, USA). Cell Counting Kit-8 (CCK-8) was purchased from Dojindo Molecular Technologies, Inc. (Kumamoto, Japan). IL-8 (cat. no. 88-8086-88) and TNF- $\alpha$ (cat. no. 88-7346-88) enzyme-linked immunosorbent assay (ELISA) kits were obtained from eBioscience, Inc., (San Diego, CA, USA). Anti-GAPDH (cat. no. 5174), anti-phospho (p) p38 MAPK (cat. no. 4511), anti-pERK1/2 (cat. no. 9154), anti-pJNK (cat. no. 4668) and anti- $\beta$-actin (cat. no. 12413) antibodies were supplied by Cell Signaling Technology, Inc. (Danvers, MA, USA). Enhanced chemiluminescence (ECL) Prime kit was purchased from GE Healthcare Life Sciences (Chalfont, UK). SB202190, SP600125 and PD98059 were purchased from Selleck Chemicals (Houston, TX, USA). Anisomycin was obtained from EMD Millipore (Billerica, MA, USA).

Cell culture. MGC 803 cells obtained from the Type Culture Collection of Chinese Academy of Sciences (Shanghai, China) were cultured in RMPI 1640 medium (Thermo Fisher Scientific, Inc.), supplemented with $10 \%$ FBS. The cells were cultured at $37^{\circ} \mathrm{C}$ in a humidified incubator with $5 \% \mathrm{CO}_{2}$.

Proliferation assay. Cells were seeded in $100 \mu \mathrm{l}$ medium at $1.0 \times 10^{4}$ cells $/ \mathrm{ml}$ in 96 -well culture plates and cultured overnight. Following pre-incubation with or without inhibitors of p38 MAPK (25 $\mu$ M SB202190), ERK1/2 (20 $\mu$ M PD98059), JNK $(20 \mu \mathrm{MSP} 600125)$ and the activator of MAPKs $(0.05 \mu \mathrm{g} / \mathrm{ml}$ anisomycin) for $1 \mathrm{~h}$, cells were treated with BER $(0,7.5,15,30$ and $60 \mu \mathrm{M})$ for 24 or $48 \mathrm{~h}$. The medium was then removed and replaced with equal volume of fresh medium with additional $10 \mu \mathrm{l} \mathrm{CCK}-8$ solution and incubated at $37^{\circ} \mathrm{C}$ for $20 \mathrm{~min}$. Absorbance of the dissolved solutions was detected at $450 \mathrm{~nm}$ using a Varioskan Flash microplate reader (Thermo Fisher Scientific, Inc.). Cell viability rate (\%) was calculated as follows: (Absorbance of drug-treated sample / absorbance of control sample) x100.

ELISA assay. For in vitro experiments, MGC 803 cells were seeded in 96-well culture plates and cultured overnight. Following treatment with $\operatorname{BER}(0,15,30$ and $60 \mu \mathrm{M})$ for $48 \mathrm{~h}$, culture medium was collected and subjected to IL- 8 and TNF- $\alpha$ ELISA assay using the respective kits. To identify the involvement of MAPKs in modulation of IL-8 expression, the cells were pre-treated with SB202190 (25 $\mu \mathrm{M})$, SP600125 (20 $\mu \mathrm{M})$, PD98059 $(20 \mu \mathrm{M})$ and anisomycin $(0.25 \mu \mathrm{g} / \mathrm{ml})$ for $1 \mathrm{~h}$, then treated with or without BER $(60 \mu \mathrm{M})$ for 24 or $48 \mathrm{~h}$. The culture medium was collected for IL-8 ELISA.

For in vivo experiments, serum and the supernatant of tumor homogenates from nude mice xenografts were used for IL-8 ELISA.

Western blotting analysis. Cells or tumor tissues were lysed with CelLytic MT Cell Lysis Reagent (Sigma-Aldrich, St. Louis, MO, USA) and sonicated three times, each for $15 \mathrm{sec}$. The lysate was centrifuged at $14,000 \mathrm{x} \mathrm{g}$ for $15 \mathrm{~min}$ at $4^{\circ} \mathrm{C}$ and the supernatant was collected. Protein concentration was determined by the bicinchoninic acid method. Protein samples $(30 \mu \mathrm{g})$ were separated by SDS-PAGE and transferred onto polyvinylidene difluoride (PVDF) membrane using the wet transfer method. Then, PVDF membranes were blocked with $5 \%$ non-fat milk solution at room temperature for $1 \mathrm{~h}$ and incubated with the different primary antibodies (anti-GAPDH, 1:5,000; anti-p-p 38, 1:1,000; anti-p-ERK, 1:1,000; anti-p-JNK, 1:1,000; and anti- $\beta$-actin, 1:2,000) overnight at $4^{\circ} \mathrm{C}$. After washing with $1 \mathrm{X}$ phosphate-buffered saline Tween 20 (PBST), PVDF membranes were incubated with the respective secondary antibodies (1:5,000; cat. no. 111-035-003; Jackson ImmunoResearch Laboratories, Inc., West Grove, PA, USA) for $1 \mathrm{~h}$ at room temperature. The protein bands were visualized with the ECL Prime kit and X-ray films.

Reverse transcription-quantitative polymerase chain reaction (RT-qPCR). Total RNA was extracted from the MGC 803 cells by using TRIzol reagent. RT was performed using a PrimeScript RT reagent kit (Takara Biotechnology Co., Ltd., Dalian, China). Forward and reverse primers used for qPCR are presented in Table I. qPCR reactions were performed using SYBR Premix Ex Taq (Takara Biotechnology Co., Ltd.) under the following cycling conditions: Initial step of $95^{\circ} \mathrm{C}$ for $30 \mathrm{sec}$; followed by $95^{\circ} \mathrm{C}$ for $5 \mathrm{sec}$ and $60^{\circ} \mathrm{C}$ for $34 \mathrm{sec}$ for 40 cycles; with final steps of $95^{\circ} \mathrm{C}$ for $15 \mathrm{sec}, 60^{\circ} \mathrm{C}$ for $1 \mathrm{~min}$ and $95^{\circ} \mathrm{C}$ for $15 \mathrm{sec}$. The relative expression level of IL- 8 was normalized to that of GAPDH in the same sample. Relative expression of target genes was normalized to GAPDH, analyzed by $2^{-\Delta \Delta \mathrm{Cq}}$ method (21) and presented as a ratio compared with the control.

Tumor xenograft model in nude mice. Thirty 4-week-old male BALB/C nude mice were purchased from Shanghai SLAC Laboratory Animal Co., Ltd. (Shanghai, China; license no. SCXK 2014-0008). The tumor xenograft model was established by subcutaneous injection of MGC 803 cells $\left(5 \times 10^{6}\right.$ cells in $200 \mu \mathrm{l}$ PBS) into the right flank of the mouse. Animals bearing tumors were randomly divided into five groups $(n=6)$ as follows: i) Control group; ii) EVO group (45 mg/kg); iii) BER group (15 mg/kg); iv) BER + EVO group (EVO $45 \mathrm{mg} / \mathrm{kg}$, BER $15 \mathrm{mg} / \mathrm{kg}$ ) and v) 5-Fu group (25 mg/kg). Prior to injection of the MGC 803 cells, mice were orally administrated with BER, EVO or 5-Fu, and administration was continued for 23 days. Body weight and two perpendicular tumor diameters (width, a; length, b) were recorded every 4 days. The tumor volume was calculated as $\mathrm{ab}^{2} / 2$. Following completion of treatment, the mice were sacrificed using $1 \%$ pentobarbital sodium (DingGuo Biotech Co., Ltd., Shanghai, China). The tumors 
Table I. Sequences of primers used for reverse transcription-quantitative polymerase chain reaction.

\begin{tabular}{lcc}
\hline Gene & Forward primer & Reverse primer \\
\hline GAPDH & GCACCGTCAAGGCTGAGAAC & TGGTGAAGACGCCAGTGGA \\
Interleukin-8 & CATACTCCAAACCTTTCCACC & AAACTTCTCCACAACCCTCTG
\end{tabular}
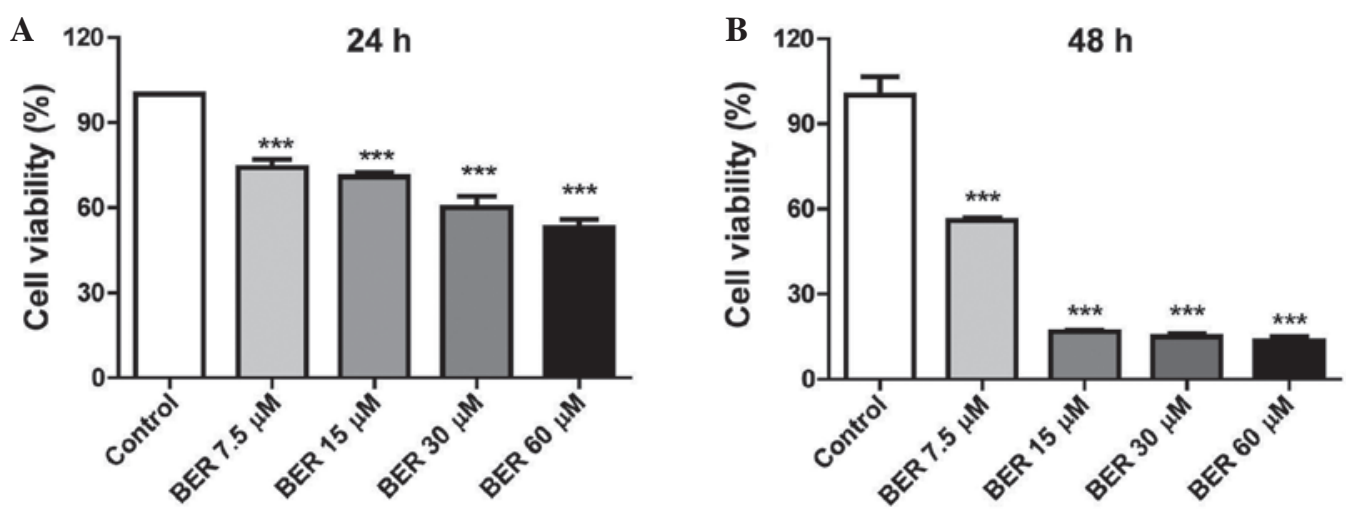

Figure 1. Effect of BER on MGC 803 cell viability. BER treatment for (A) $24 \mathrm{~h}$ and (B) $48 \mathrm{~h}$ decreased cell viability, which was measured by Cell Counting Kit- 8 assay. Data are presented as the mean \pm standard error. ${ }^{* * * *} \mathrm{P}<0.001$ vs. control. BER; berberine hydrochloride.

were dissected, weighed, and stored at $-80^{\circ} \mathrm{C}$ for use in ELISA and western blotting.

All animal experiments were performed according to the protocols approved by Animal Care and Use Committee of Shanghai University of Traditional Chinese Medicine, (Shanghai, China) which complies with international rules and policies. All efforts were made to minimize suffering and reduce the number of animals used.

Statistical analysis. Values are presented as the mean \pm standard error. Differences among groups were analyzed by one-way analysis of variance with Newman-Keuls test using Prism software (version 5; GraphPad Software, Inc., La Jolla, CA, USA). $\mathrm{P}<0.05$ was considered to indicate a statistically significant difference.

\section{Results}

BER suppresses proliferation of MGC 803 cells in vitro. BER demonstrated an inhibitory effect on the viability of MGC 803 cells in a dose- and time-dependent manner. As demonstrated in Fig. 1, BER treatment (7.5-60 $\mu \mathrm{M})$ significantly decreased the cell viability compared with the control $(\mathrm{P}<0.001)$ at 24 and $48 \mathrm{~h}$. Furthermore, prolonged BER treatment (48 h) led to increased injury to the MGC 803 cells as demonstrated by the reduced cell viability.

BER inactivates MAPK pathways in MGC 803 cells. To determine the association between MAPK signaling pathways, including p38 MAPK, ERK1/2 and JNK pathway, and cell survival of MGC 803 cells, western blot analysis was performed. As demonstrated in Fig. 2, BER treatment at 15, 30 and $60 \mu \mathrm{M}$ for 24 and $48 \mathrm{~h}$ reduced the phosphorylation of p38 MAPK, ERK1/2 and JNK in a dose- and time-dependent manner.

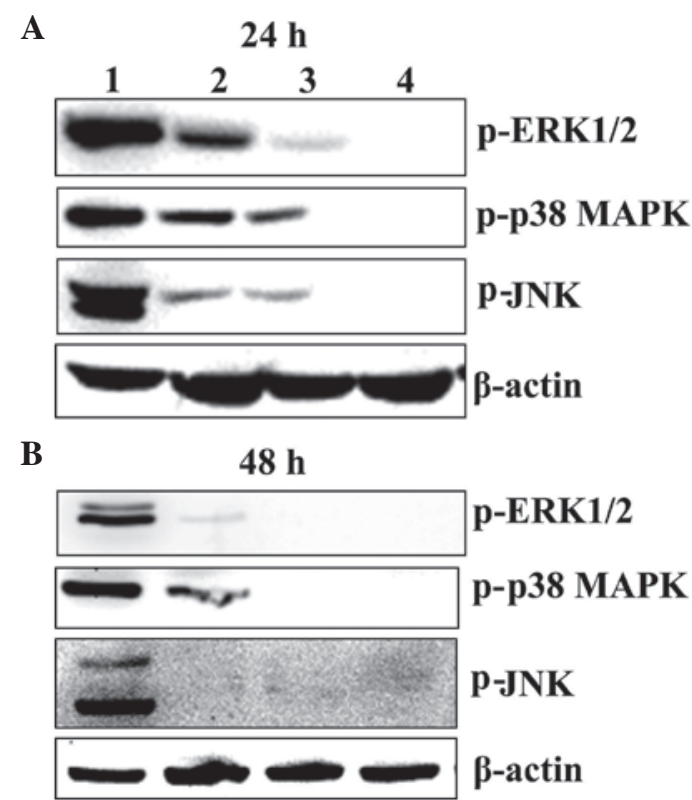

Figure 2. Effect of BER on the phosphorylation of p38 MAPK, ERK1/2 and JNK in MGC 803 cells. BER treatment for (A) $24 \mathrm{~h}$ and (B) $48 \mathrm{~h}$ inhibited intracellular phosphorylation of p38 MAPK, ERK1/2 and JNK, as measured by western blotting. 1: Control; 2: $15 \mu \mathrm{M}$ BER; 3: $30 \mu \mathrm{M}$ BER; $60 \mu \mathrm{M}$ BER. BER, berberine hydrochloride; MAPK, mitogen-activated protein kinase; ERK, extracellular signal-regulated kinase; JNK, c-Jun N-terminal kinase.

In order to further clarify the importance of MAPK signaling in the cell proliferation, and whether BER inhibited cell proliferation of gastric cancer cells via deactivating MAPKs, the inhibitors of p38 MAPK (SB202190), JNK (SP600125) and ERK1/2 (PD98059) were used in a CCK-8 assay. As demonstrated in Fig. 3A, in MGC 803 cells, these inhibitors enhanced the inhibitory effect of BER on cell proliferation compared with BER treatment $(\mathrm{P}<0.001, \mathrm{P}<0.001$ and 
A

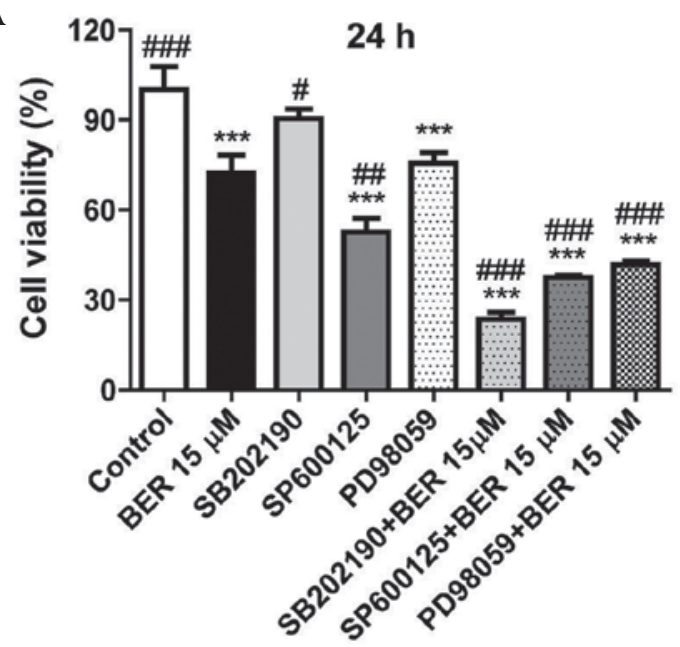

C

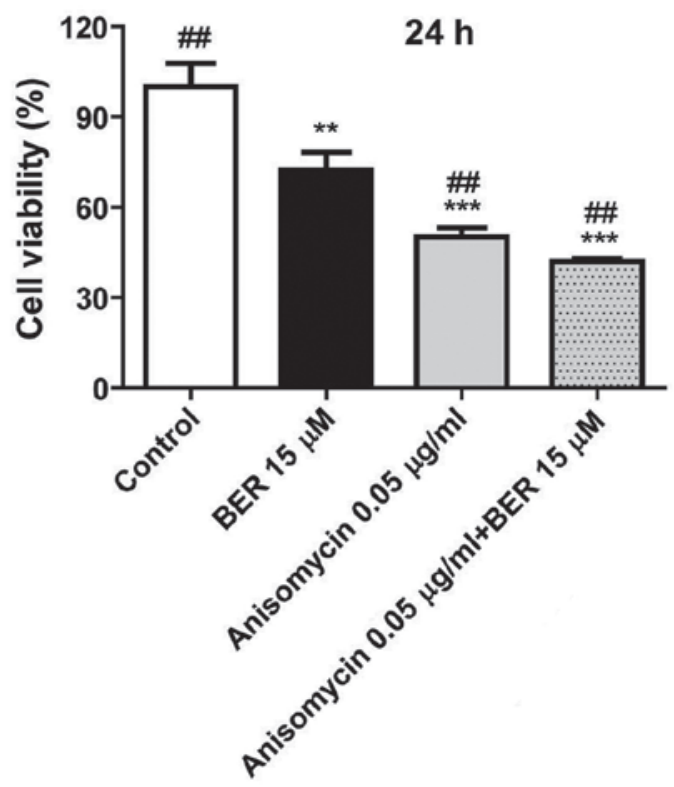

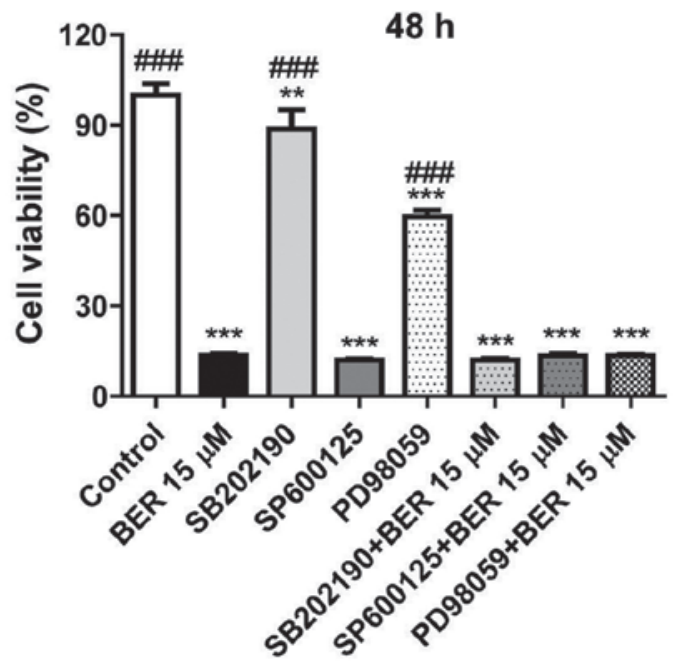

D

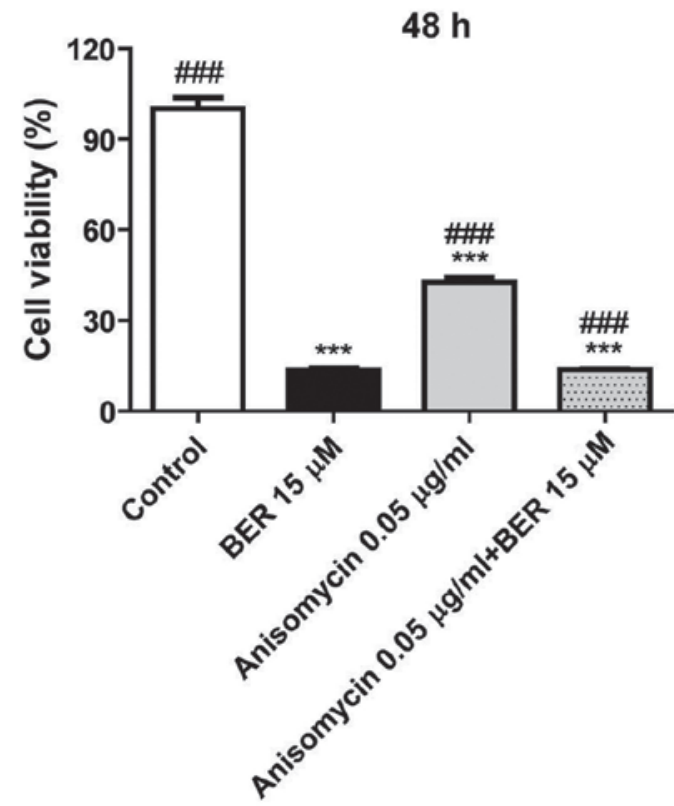

Figure 3. MAPKs are involved in the inhibitory effect of BER on cell proliferation. (A) Cell viability was detected by Cell Counting Kit-8 assay following treatment with $15 \mu \mathrm{M}$ BER, $25 \mu \mathrm{M}$ SB202190 (p38 MAPK inhibitor), $20 \mu \mathrm{M}$ SP600125 (JNK inhibitor) and $20 \mu \mathrm{M}$ PD98059 (extracellular signal-regulated kinase $1 / 2$ inhibitor) for $24 \mathrm{~h}$ and (B) the same conditions for $48 \mathrm{~h}$. (C) Cell viability was detected by Cell Counting Kit- 8 assay following treatment with $15 \mu \mathrm{M}$ BER and $0.05 \mu \mathrm{g} / \mathrm{ml}$ anisomycin (p38 MAPK and JNK activator) for $24 \mathrm{~h}$ and (D) under the same conditions for $48 \mathrm{~h}$. Data are presented as the mean \pm standard error. ${ }^{* *} \mathrm{P}<0.01,{ }^{* * *} \mathrm{P}<0.001$ vs. control. ${ }^{\#} \mathrm{P}<0.05,{ }^{\# \#} \mathrm{P}<0.01,{ }^{\# \# "} \mathrm{P}<0.001$ vs. BER $15 \mu \mathrm{M}$. BER, berberine hydrochloride; MAPK, mitogen-activated protein kinase; JNK, c-Jun N-terminal kinase.

$\mathrm{P}<0.05)$. Notably, anisomycin, an activator of p38 MAPK and JNK, also significantly reduced cell viability of MGC 803 cells compared with controls (Fig. 3C). As demonstrated in Fig. 3B and D, as BER treatment for $48 \mathrm{~h}$ killed the majority of the cancer cells, combination of BER with the inhibitors or activator of MAPKs did not demonstrate a synergistic effect at this time point.

BER decreases IL-8 secretion and gene expression levels in $M G C 803$ cells. In previous research, BER was demonstrated to inhibit IL-8 expression in a dose- and time-dependent manner in AGS and MDA-MB-231 cells $(10,12)$. However, whether the inhibitory effect of BER on IL-8 expression is cell-type specific remains unclear. The present study demonstrated that BER also reduces the secretion $(\mathrm{P}<0.001 ;$ Fig. $4 \mathrm{~A})$ and gene expression levels $(\mathrm{P}<0.001$; Fig. 4B) of IL-8 in MGC 803 cells compared with controls. Further investigation demonstrated that anisomycin significantly increased the secretion of IL-8 and reduced cell viability of MGC 803 cells compared with controls $(\mathrm{P}<0.001)$, which was completely abolished by combination with BER treatment $(\mathrm{P}<0.001$; Fig. $4 \mathrm{C}$ and $\mathrm{D})$. Furthermore, as demonstrated in Fig. 4E, treatment with SB202190, SP600125 or PD98059 significantly decreased IL-8 secretion in MGC 803 cells compared with controls $(\mathrm{P}<0.001)$. However, BER did not alter TNF- $\alpha$ production of the cells (Fig. 4F). Thus, the results of the present study indicated that BER specifically affects IL-8 production in MGC 803 cells.

BER inhibits tumor development from MGC 803 cells in vivo. To examine the anti-tumor effect of BER in vivo, a human gastric cancer xenograft model was used in BALB/C nude mice. In nude mice transplanted with MGC 803 cells were treated 
A

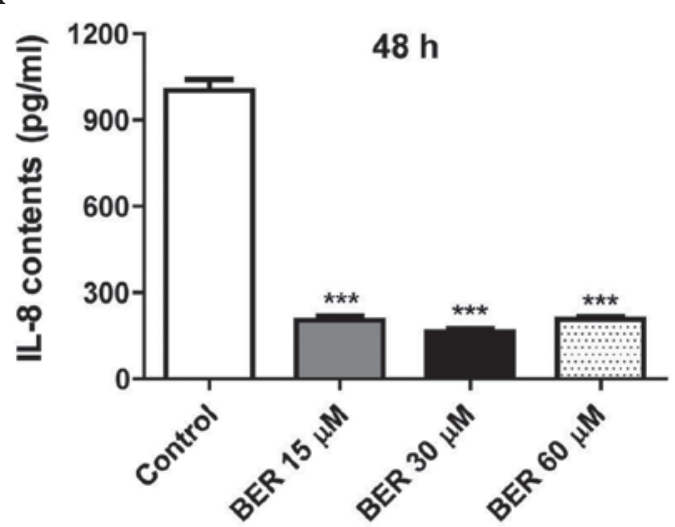

C

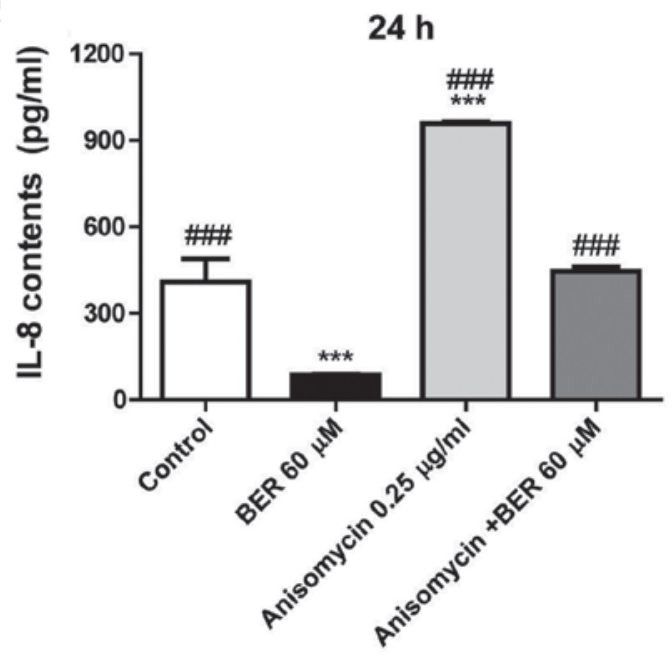

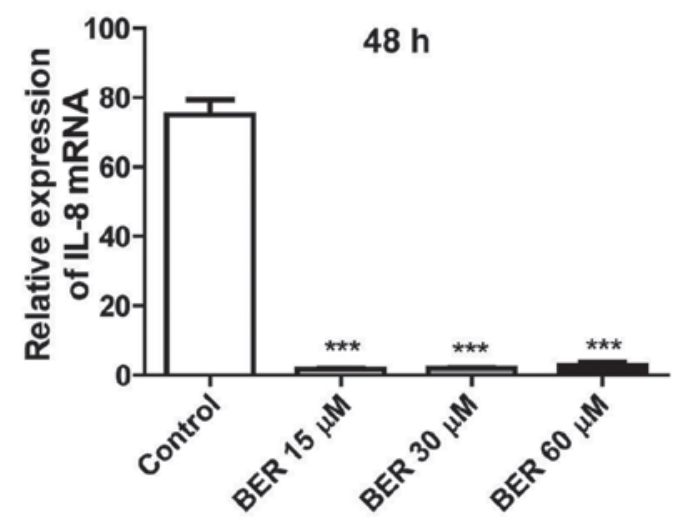

D

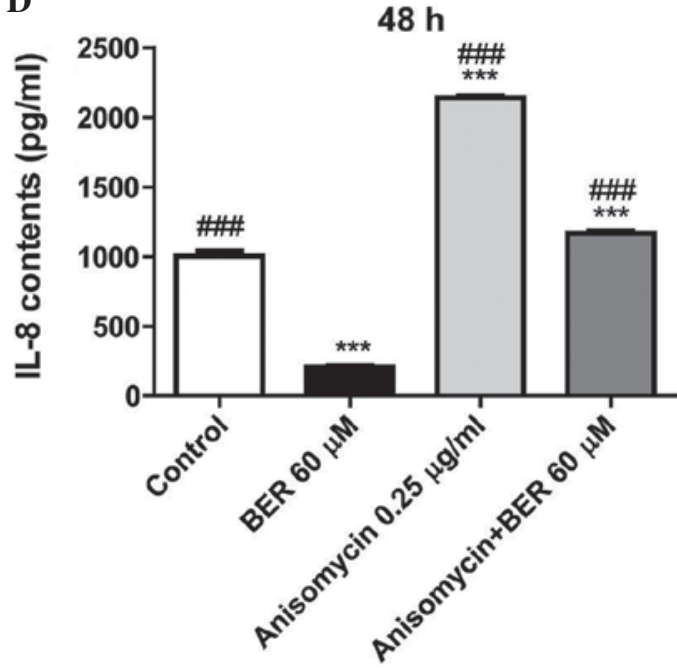

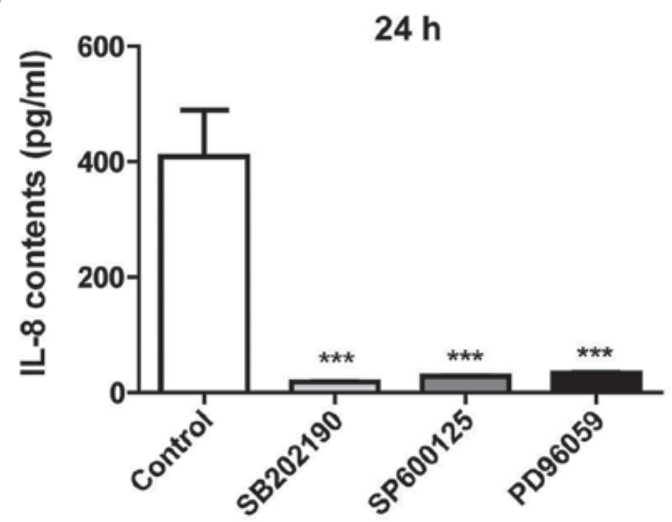

F

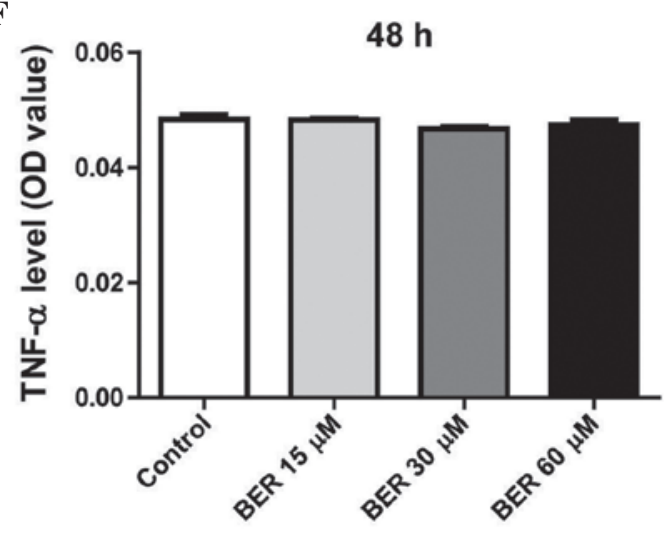

Figure 4. Effect of BER on IL-8 gene expression and secretion of MGC 803 cells. (A) BER treatment (60 $\mu \mathrm{M})$ for 48 h decreased IL-8 secretion. (B) BER treatment $(60 \mu \mathrm{M})$ for $48 \mathrm{~h}$ reduced the mRNA levels of IL-8. (C) BER treatment $(60 \mu \mathrm{M})$ for $24 \mathrm{~h}$ and (D) $48 \mathrm{~h}$ inhibited IL-8 expression through deactivating MAPKs, shown using activator of p38 MAPK and JNK, anisomycin $(0.25 \mu \mathrm{g} / \mathrm{ml})$. (E) Inhibitors of p38 MAPK (SB202190), JNK (SP600125) and extracellular signal-regulated kinase 1/2 (PD98059) prevented IL-8 secretion after treatment with BER $(60 \mu \mathrm{M})$ for $24 \mathrm{~h}$. (F) BER treatment $(60 \mu \mathrm{M})$ for $48 \mathrm{~h}$ did not affect TNF- $\alpha$ secretion. Data are presented as the mean \pm standard error. ${ }^{* * * *} \mathrm{P}<0.001$ vs. control; ${ }^{\# \#} \mathrm{P}<0.001$ vs. BER $60 \mu \mathrm{M}$. BER, berberine hydrochloride; MAPK, mitogen-activated protein kinase; JNK, c-Jun N-terminal kinase.

with the drugs for 23 days. $5-\mathrm{Fu}(25 \mathrm{mg} / \mathrm{kg})$, was administered as a positive control and significantly prevented the growth of tumor (Fig. 5), with the tumor weight and volume of mice in this group significantly reduced compared with the control group $(\mathrm{P}<0.001)$. However, long-term treatment with 5-Fu led to a significant reduction in the body weight compared with control mice $(\mathrm{P}<0.001)$. By contrast, BER $(15 \mathrm{mg} / \mathrm{kg}$ daily $)$ significantly reduced the tumor weight and tumor volume compared with controls $(\mathrm{P}<0.05$ and $\mathrm{P}<0.001$, respectively), and also did not lead to body weight loss. EVO treatment also 
$\mathbf{A}$

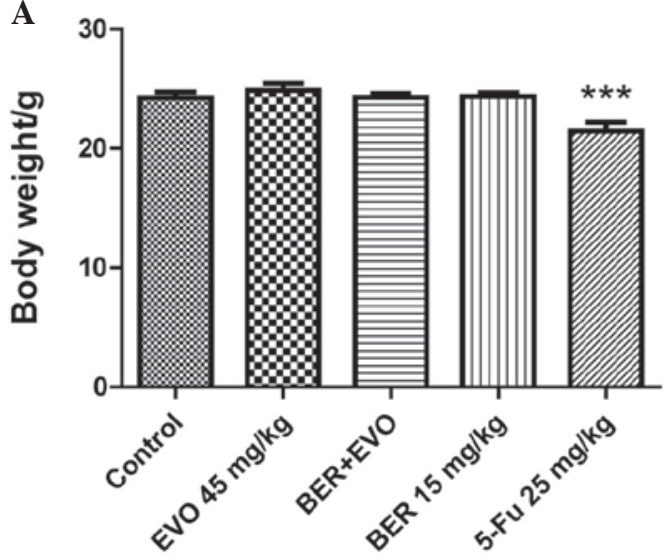

C

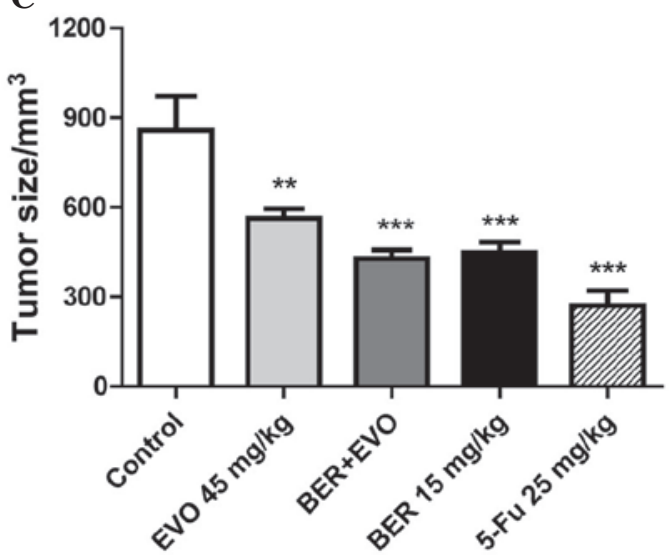

B

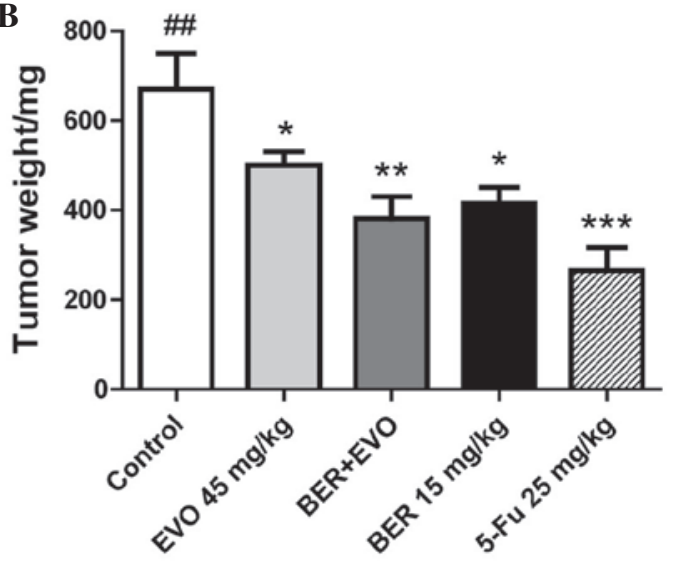

D

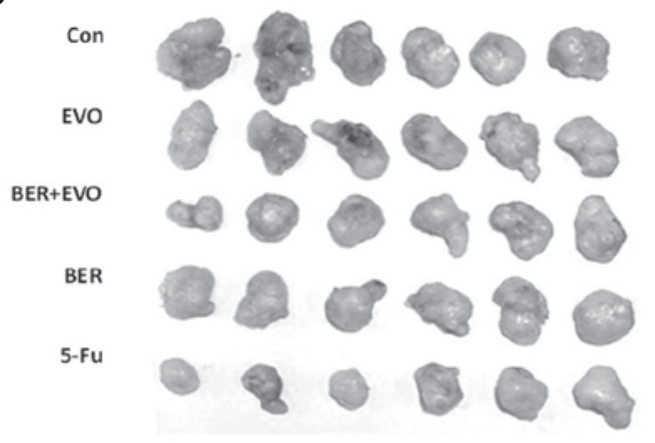

Figure 5. Effect of BER on tumor growth of human gastric cancer xenograft. (A) BER exhibited no obvious effect on body weight of nude mice, while 5-Fu induced a significant weight loss. BER, EVO, 5-Fu and BER + EVO reduced the (B) tumor weight and (C) tumor size following treatment for 23 days, however, there was no obvious synergistic effect between BER and EVO. (D) Comparison of xenograft tumors excised from the mice treated with control, EVO, BER + EVO, BER and 5-Fu. Data are presented as the mean \pm standard error. ${ }^{*} \mathrm{P}<0.05,{ }^{* * *} \mathrm{P}<0.01,{ }^{* * * *} \mathrm{P}<0.001$ vs. control; ${ }^{\# \#} \mathrm{P}<0.01$ vs. BER $+\mathrm{EVO}$. BER, berberine hydrochloride; EVO, evodiamine; 5-Fu, fluorouracil.

A

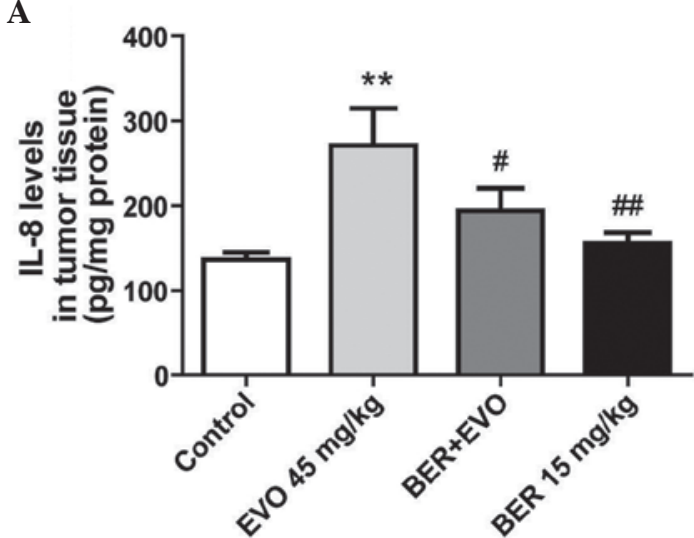

B

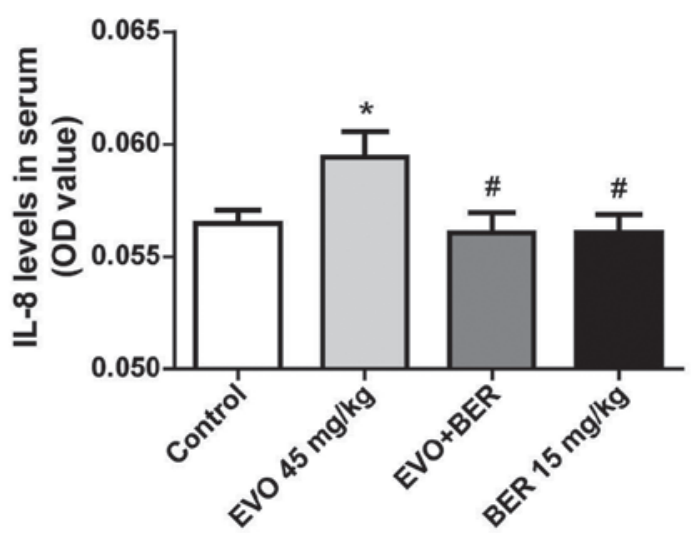

Figure 6. Effect of BER on IL-8 secretion in nude mice with gastric cancer. (A) BER decreased IL-8 level in tumor tissue measured by ELISA assay (B), BER decreased IL-8 secretion in serum measured by ELISA assay. Data are presented as the mean \pm standard error. ${ }^{*} \mathrm{P}<0.05,{ }^{* *} \mathrm{P}<0.01$ vs. control. ${ }^{\#} \mathrm{P}<0.05$, ${ }^{, \#} \mathrm{P}<0.01$ vs. EVO $45 \mathrm{mg} / \mathrm{kg}$. ELISA, enzyme-linked immunosorbent assay; IL-8, interleukin-8; OD, optical density; BER, berberine hydrochloride; EVO, evodiamine.

significantly inhibited tumor weight and size compared with controls $(\mathrm{P}<0.05$ and $\mathrm{P}<0.01$, respectively). Co-administration of EVO and BER demonstrated a trend to inhibit tumor weight and volume compared with BER or EVO alone, but without a statistically significant difference. Both EVO and combination of BER and EVO demonstrated no significant effect on body weight.

BER inhibits upregulation of IL-8 induced by EVO in nude mice xenografted with MGC 803 cells. In a previous study, 
EVO was demonstrated to upregulate IL-8 expression in AGS cells in vitro (10), whether it induces IL-8 expression in vivo remains to be determined. In the present study, EVO, the alkaloid from Evodia fructus, significantly suppressed MGC 803 tumor development in nude mice compared with controls (Fig. 5), but increased IL-8 production in tumor tissue $(\mathrm{P}<0.01$; Fig. 6A) and serum $(\mathrm{P}<0.05 ;$ Fig. 6B). BER treatment alone, or in combination with EVO significantly attenuated the increase of IL-8 in tumor tissue and serum compared with EVO treatment $(\mathrm{P}<0.01$ and $\mathrm{P}<0.05$, respectively).

BER inactivates MAPKs in the tumor tissue of nude mice xenografted with MGC 803 cells. Following treatment with BER at a dose of $15 \mathrm{mg} / \mathrm{kg}$ for 23 days, the phosphorylation levels of p38 MAPK, ERK1/2 and JNK in tumor tissue was significantly reduced compared with controls (Fig. 7). By contrast, EVO alone did not alleviate the phosphorylation of p38 MAPK, ERK1/2 and JNK compared with control levels. However, co-treatment with BER and EVO markedly reduced the phosphorylation of the MAPKs in tumor tissues compared with control and EVO-treated nude mice.

\section{Discussion}

Previous studies have demonstrated the inhibitory effect of BER on the proliferation of cancer cells, and that the effect is predominantly mediated via the inactivation of the phosphatidylinositol 3-kinase/AKT serine/threonine kinase 1 signaling pathway $(12,13)$. MAPK pathways have also previously been indicated to be involved in the anti-cancer effect of BER, however reports vary depending on the cancer cell type $(12,16-20)$. Although BER has previously been demonstrated to exert anti-tumorigenesis functions in various gastric cancer cell lines, including SGC 7901, BGC 823, AGS, SNU-5, SC-M1 and NUGC-3 cells $(10,13,22,23)$, its effect on cell viability, IL-8 expression and MAPK signaling in MGC 803 cells has not been previously investigated. In the current study, BER was demonstrated to significantly decrease the cell viability of MGC 803 cells in a dose- and time-dependent manner. Further analysis demonstrated that the phosphorylation of p38 MAPK, ERK1/2 and JNK were inhibited by BER even at a low concentration $(15 \mu \mathrm{M})$. Using inhibitors of p38 MAPK (SB202190), ERK1/2 (PD98059) and JNK (SP600125), the present study demonstrated that the downregulated phosphorylation of p38 MAPK, ERK1/2 and JNK were involved in the inhibitory effect of BER on the cell viability of MGC 803 cells. Considering the difference between in vitro and in vivo settings, the effect of BER on gastric tumors developed from xenografted MGC 803 cells was also evaluated. The results demonstrated that $15 \mathrm{mg} / \mathrm{kg}$ BER significantly inhibited the tumor growth and reduced the phosphorylation of p38 MAPK, ERK1/2 and JNK. Thus, inactivation of MAPK signaling is indeed involved in the anti-tumor activity of BER on MGC 803 cells in vitro and in vivo. Furthermore, compared with 5 -Fu, BER inhibited tumor growth without affecting body weight, which is a general side-effect of chemotherapeutic drugs.

It is well established that various chemotherapeutic agents induce upregulation of IL-8 levels in tumor cells, which is closely associated with chemotherapy resistance

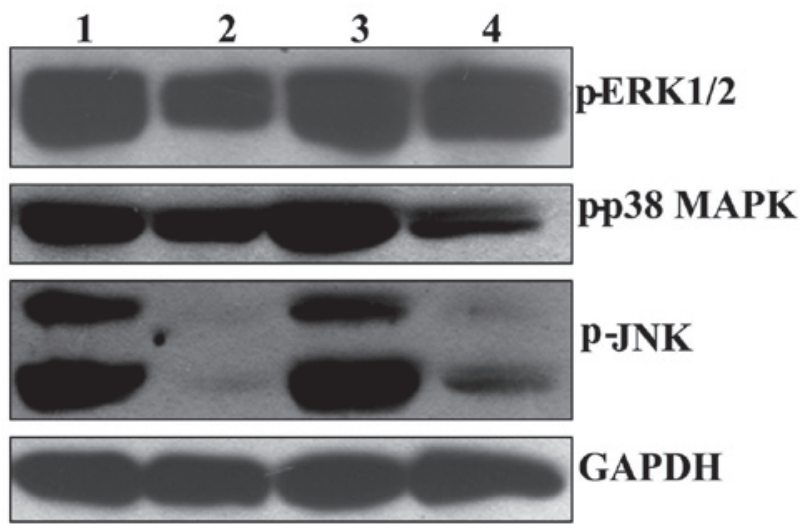

Figure 7. Effect of BER on phosphorylation of p38 MAPK, ERK1/2 and JNK in tumor tissues of nude mice bearing gastric cancer. After treatment with drugs for 23 days, the mice were sacrificed and the tumor were dissected, homogenized and subjected to western blotting assay. 1: Control; 2: BER, $15 \mathrm{mg} / \mathrm{kg}$; 3: EVO, $45 \mathrm{mg} / \mathrm{kg}$; 4: EVO+BER. MAPK, mitogen-activated protein kinase; ERK, extracellular signal-regulated kinase; JNK, c-Jun $\mathrm{N}$-terminal kinase; BER, berberine hydrochloride; EVO, evodiamine.

and cancer metastasis $(7,9,10,12,24-30)$. Overexpression of IL-8 in cancer cells is known to be important for the tumor microenvironment via binding to CXC motif chemokine receptor 1 (CXCR1) and CXCR2 receptors on tumor cells, neutrophils/tumor-associated macrophages and endothelial cells, and promotes angiogenesis and metastasis $(7,9,24,28,31)$. By contrast, depletion of IL-8 induces cell cycle arrest and increases the efficacy of chemotherapeutic agents in breast cancer cells (32). Thus, chemotherapeutic agents with an inhibitory effect on IL-8 production may have increased efficacy. In the present investigation, BER significantly decreased IL-8 secretion in MGC 803 cells in vitro and in vivo, demonstrating its safety and efficacy in inhibiting gastric cancer cells.

MAPKs have previously been demonstrated to be involved in the modulation of IL-8 production $(25,28,33)$. In accordance with the previous reports, the current results indicated that activation of the ERK1/2, JNK and p38 MAPK signaling pathways was closely associated with constitutive IL-8 secretion in MGC 803 cells. Additionally, inhibitors of p38 MAPK, JNK and ERK significantly decreased IL-8 expression in MGC 803 cells. Whereas, when the MAPK agonist, anisomycin, was applied, IL-8 secretion was upregulated. BER, similarly to the MAPK inhibitors, reduced the phosphorylation of ERK1/2, JNK and p38 MAPK, and counteracted the increased IL-8 secretion induced by anisomycin. Activation of MAPKs by anisomycin also decreased cell viability of MGC 803 cells. However, in accordance with the findings of the current study, curcumin has previously been reported to induce apoptosis through activation of ERK1/2 in AGS cells (34), whereas apoptosis was enhanced by capsaicin through inhibition of MAPKs in AGS cells (35). These results reflect the complex functions of MAPKs in cancer cells.

In a previous study in AGS cells, EVO was demonstrated to significantly enhance IL-8 expression, the effect of which was counteracted by BER (10). Consistent with its in vitro effects, the results of the current study demonstrated that in addition to the inhibition of tumor growth, EVO upregulated 
IL-8 secretion in serum and tumor tissue of tumor xenografted nude mice, which was also inhibited by BER. As it is not constitutively expressed in mice (36), the levels of IL-8 in nude mouse serum secreted from tumor tissue was relatively low, the optical density value of which was beyond the limit of detection of the ELISA kit. Compared with control mice, EVO-treated mice did not demonstrate significant changes in the levels of phosphorylated p38 MAPK, ERK1/2 and JNK. However, when BER was added, the level of phosphorylation of MAPKs in MGC 803 cell-derived tumors was markedly reduced. These results indicated that co-administration of EVO with BER may be a safer therapy for gastric cancer without reduction of its efficacy.

In conclusion, BER reduced the growth of MGC 803 cells and IL-8 expression levels in vitro and in vivo, which was associated with deactivation of p38 MAPK, ERK1/2 and JNK signaling pathways. Furthermore, BER significantly counteracted the upregulation of IL-8 production induced by EVO in vivo. The findings of the present study demonstrated the potential safety and efficacy of BER in the clinical therapy of gastric cancer.

\section{Acknowledgements}

This work was supported by the Educational Commission of Shanghai of China (grant no. 2012JW19), Key Research Innovation Project (grant no. 13ZZ099), Key Project from Department of Education of China (grant no. 20123107130002), Shanghai Eastern Scholar Program (grant no. 2013-59) and Shanghai E-research Institute of Bioactive Constituent in TCM plan.

\section{References}

1. Macdonald JS: Treatment of localized gastric cancer. Semin Oncol 31: 566-573, 2004.

2. Ferlay J, Shin HR, Bray F, Forman D, Mathers C and Parkin DM: Estimates of worldwide burden of cancer in 2008: GLOBOCAN 2008. Int J Cancer 127: 2893-2917, 2010.

3. Lurje G, Husain H, Power DG, Yang D, Groshen S, Pohl A, Zhang W, Ning Y, Manegold PC, EI-Khoueiry A, et al: Genetic variations in angiogenesis pathway genes associated with clinical outcome in localized gastric adenocarcinoma. Ann Oncol 21: 78-86, 2010.

4. Zheng L, Wang L, Ajani J and Xie K: Molecular basis of gastric cancer development and progression. Gastric Cancer 7: 61-77, 2004.

5. Mitchell MS and DeConti RC: Immunosuppression by 5-fluorouracil. Cancer 26: 884-889, 1970.

6. Justino PF, Melo LF, Nogueira AF, Morais CM, Mendes WO, Franco AX, Souza EP, Ribeiro RA, Souza MH and Soares PM: Regulatory role of Lactobacillus acidophilus on inflammation and gastric dysmotility in intestinal mucositis induced by 5 -fluorouracil in mice. Cancer Chemother Pharmacol 75: 559-567, 2015.

7. Kuai WX, Wang Q, Yang XZ, Zhao Y, Yu R and Tang XJ: Interleukin-8 associates with adhesion, migration, invasion and chemosensitivity of human gastric cancer cells. World J Gastroenterol 18: 979-985, 2012.

8. Bünger S, Haug U, Kelly FM, Klempt-Giessing K, Cartwright A, Posorski N, Dibbelt L, Fitzgerald SP, Bruch HP, Roblick UJ, et al: Toward standardized high-throughput serum diagnostics: Multiplex-protein array identifies IL-8 and VEGF as serum markers for colon cancer. J Biomol Screen 16: 1018-1026, 2011.

9. Ju D, Sun D, Xiu L, Meng X, Zhang C and Wei P: Interleukin-8 is associated with adhesion, migration and invasion in human gastric cancer SCG-7901 cells. Med Oncol 29: 91-99, 2012.
10. Shi HL, Wu XJ, Liu Y and Xie JQ: Berberine counteracts enhanced IL-8 expression of AGS cells induced by evodiamine. Life Sci 93: 830-839, 2013

11. Wang XN, Han X, Xu LN, Yin LH, Xu YW, Qi Y and Peng JY: Enhancement of apoptosis of human hepatocellular carcinoma SMMC-7721 cells through synergy of berberine and evodiamine. Phytomedicine 15: 1062-1068, 2008.

12. Li X, Zhao SJ, Shi HL, Qiu SP, Xie JQ, Wu H, Zhang BB, Wang ZT, Yuan JY and Wu XJ: Berberine hydrochloride IL-8 dependently inhibits invasion and IL-8-independently promotes cell apoptosis in MDA-MB-231 cells. Oncol Rep 32: 2777-2788, 2014.

13. Yi T, Zhuang L, Song G, Zhang B, Li G and Hu T: Akt signaling is associated with the berberine-induced apoptosis of human gastric cancer cells. Nutr Cancer 67: 523-531, 2015.

14. Liu X, Ji Q, Ye N, Sui H, Zhou L, Zhu H, Fan Z, Cai J and Li Q: Berberine inhibits invasion and metastasis of colorectal cancer cells via COX-2/PGE2 mediated JAK2/STAT3 signaling pathway. PLoS One 10: e0123478, 2015.

15. Shi HL, Xie JQ and Wu DZ: Effect of berberine on cell proliferation and IL-8 expression in AGS cells. Zhong Yao Yao Li Yu Lin Chuang Bian Ji Bu 28: 45-48, 2012.

16. Hsu WH, Hsieh YS, Kuo HC, Teng CY, Huang HI, Wang CJ, Yang SF, Liou YS and Kuo WH: Berberine induces apoptosis in SW620 human colonic carcinoma cells through generation of reactive oxygen species and activation of JNK/p38 MAPK and FasL. Arch Toxicol 81: 719-728, 2007.

17. Zheng F, Tang Q, Wu J, Zhao S, Liang Z, Li L, Wu W and Hann S: $\mathrm{P} 38 \alpha$ MAPK-mediated induction and interaction of FOXO3a and p53 contribute to the inhibited-growth and induced-apoptosis of human lung adenocarcinoma cells by berberine. J Exp Clin Cancer Res 33: 36, 2014.

18. Hyun MS, Hur JM, Mun YJ, Kim D and Woo WH: BBR induces apoptosis in HepG2 cell through an Akt-ASK1-ROSp38MAPKs-linked cascade. J Cell Biochem 109: 329-338, 2010.

19. Hur JM, Hyun MS, Lim SY, Lee WY and Kim D: The combination of berberine and irradiation enhances anti-cancer effects via activation of p38 MAPK pathway and ROS generation in human hepatoma cells. J Cell Biochem 107: 955-964, 2009.

20. Lu B, Hu M, Liu K and Peng J: Cytotoxicity of berberine on human cervical carcinoma HeLa cells through mitochondria, death receptor and MAPK pathways, and in-silico drug-target prediction. Toxicol In Vitro 24: 1482-1490, 2010.

21. Livak KJ and Schmittgen TD: Analysis of relative gene expression data using real-time quantitative PCR and the 2(-Delta Delta C(T)) Method. Methods 25: 402-408, 2001.

22. Lin HL, Liu TY, Wu CW and Chi CW: Berberine modulates expression of mdrl gene product and the responses of digestive track cancer cells to Paclitaxel. Br J Cancer 81: 416-422, 1999.

23. Lin JP, Yang JS, Lee JH, Hsieh WT and Chung JG: Berberine induces cell cycle arrest and apoptosis in human gastric carcinoma SNU-5 cell line. World J Gastroenterol 12: 21-28, 2006.

24. Kitadai Y, Takahashi Y, Haruma K, Naka K, Sumii K, Yokozaki H, Yasui W, Mukaida N, Ohmoto Y, Kajiyama G, et al: Transfection of interleukin-8 increases angiogenesis and tumorigenesis of human gastric carcinoma cells in nude mice. $\mathrm{Br}$ J Cancer 81: 647-653, 1999.

25. Collins TS, Lee LF and Ting JP: Paclitaxel up-regulates interleukin-8 synthesis in human lung carcinoma through an NF-kappaB-and AP-1-dependent mechanism. Cancer Immunol Immunother 49: 78-84, 2000.

26. Lev DC, Onn A, Melinkova VO, Miller C, Stone V, Ruiz M, McGary EC, Ananthaswamy HN, Price JE and Bar-Eli M: Exposure of melanoma cells to dacarbazine results in enhanced tumor growth and metastasis in vivo. J Clin Oncol 22: 2092-2100, 2004.

27. Kishida O, Miyazaki Y, Murayama Y, Ogasa M, Miyazaki T, Yamamoto T, Watabe K, Tsutsui S, Kiyohara T, Shimomura I and Shinomura Y: Gefitinib (Iressa, ZD1839) inhibits SN38-triggered EGF signals and IL-8 production in gastric cancer cells. Cancer Chemother Pharmacol 55: 584-594, 2005.

28. Waugh DJ and Wilson C: The interleukin-8 pathway in cancer. Clin Cancer Res 14: 6735-6741, 2008.

29. Britschgi A, Andraos R, Brinkhaus H, Klebba I, Romanet V, Müller U, Murakami M, Radimerski T and Bentires-Alj M: JAK2/STAT5 inhibition circumvents resistance to PI3 K/mTOR blockade: A rationale for cotargeting these pathways in metastatic breast cancer. Cancer Cell 22: 796-811, 2012. 
30. Zhang W,Feng M, Zheng G, Chen Y, Wang X,Pen B, Yin J, Yu Y and $\mathrm{He} \mathrm{Z}$ : Chemoresistance to 5-fluorouracil induces epithelial-mesenchymal transition via up-regulation of Snail in MCF7 human breast cancer cells. Biochem Biophys Res Commun 417: 679-685, 2012.

31. Matsuo Y, Ochi N, Sawai H, Yasuda A, Takahashi H, Funahashi H, Takeyama H, Tong Z and Guha S: CXCL8/IL-8 and CXCL12/SDF-1alpha co-operatively promote invasiveness and angiogenesis in pancreatic cancer. Int J Cancer 124: 853-861, 2009.

32. Shao N, Chen LH, Ye RY, Lin Y and Wang SM: The depletion of interleukin-8 causes cell cycle arrest and increases the efficacy of docetaxel in breast cancer cells. Biochem Biophys Res Commun 431: 535-541, 2013.

33. Bezzerri V, Borgatti M, Finotti A, Tamanini A, Gambari R and Cabrini G: Mapping the transcriptional machinery of the IL-8 gene in human bronchial epithelial cells. J Immunol 187: 6069-6081, 2011.
34. Cao AL, Tang QF, Zhou WC, Qiu YY, Hu SJ and Yin PH: Ras/ERK signaling pathway is involved in curcumin-induced cell cycle arrest and apoptosis in human gastric carcinoma AGS cells. J Asian Nat Prod Res 17: 56-63, 2015.

35. Park SY, Kim JY, Lee SM, Jun CH, Cho SB, Park CH, Joo YE, Kim HS, Choi SK and Rew JS: Capsaicin induces apoptosis and modulates MAPK signaling in human gastric cancer cells. Mol Med Rep 9: 499-502, 2014.

36. Asfaha S, Dubeykovskiy AN, Tomita H, Yang X, Stokes S, Shibata W, Friedman RA, Ariyama H, Dubeykovskaya ZA, Muthupalani S, et al: Mice that express human interleukin-8 have increased mobilization of immature myeloid cells, which exacerbates inflammation and accelerates colon carcinogenesis. Gastroenterology 144: 155-166, 2013. 